\title{
Infrared homing head for rotating missiles
}

\author{
J. Piotrowski, W. Swiderski and D. Szabra
}

\author{
Military Institute of Armament Technology \\ 05-220 Zielonka, Prymasa Wyszynskiego 7 Str, Poland \\ zak13@witu.mil.pl
}

\begin{abstract}
:
A simple infrared system operating without any mechanic-optical scanners that can be used in homing heads of rotating missiles is reported. Space scanning is achieved by missile rotation for the tangential direction scan and an electronic scan of linear array in a dedicated IR focal plane as shown in Fig. 1. Full space scanning is achieved at each rotation of the missile.

The scanning velocity is proportional to the distance of each array element to the optical axis of the system. This results in increased bandwidth, deteriorating of $\mathrm{S} / \mathrm{N}$ ratio, and in consequence, in reduced thermal resolution for the outer detectors.

Fig. 2 shows more advanced solutions in which the negative consequences of increased scanning velocity are compensated by an appropriate increase of number of detector elements operating in the TDI mode.

The probability of low temperature target detection is evaluated basing on the Johnson method as a function of detector detectivity, array geometry, optics parameters, target range and other parameters.

We have shown that useful performance of the simple homing heads can be obtained using LWIR and MWIR $\mathrm{Hg}_{1-\mathrm{x}} \mathrm{Cd}_{\mathrm{x}}$ Te photodetectors operating at near room temperatures. Such devices have been developed at VIGO SYSTEM SA. They are manufactured using complex $\mathrm{Hg}_{1-x} \mathrm{Cd}_{x} \mathrm{Te}$ heterostructures grown by the low temperature epitaxial techniques. The performance of the devices has been improved using immersion microlenses, optical resonant cavities and other solutions.
\end{abstract}

\section{Introduction}

One of the most important tendencies in military technique is the development of smart ammunition. Such ammunition has huge advantages over the conventional one. The smart ammunition is a new type of weapon with possible independent detection and attacking of target after its firing. The beginning of smart ammunition became possible with development of Automatic Targets Recognition systems (ATR) [1, 2, 3].

Infrared imaging is widely used for the ATR systems. The guidance of missiles is comparatively easy for small-sized targets with intensive radiation against the homogeneous background, much more difficult for objects with low contrast against complex and changing in time background. Detection, recognition and identification requires a more detailed analysis of observed image. This can be achieved with costly 2D imaging systems and automatic pattern recognition systems.

Additional complication is the missile rotation that is frequently used for the stabilization of missile trajectory. The rotation presents a significant challenge for space imaging $[4,5]$ disturbing the operation of conventional mechanical and electronic scanning systems. 
A simple solution of the problem is to employ the missile rotation for space scanning. This can be achieved with a simple imaging system with linear detector array situated radially in the image plane of a rotating missile homing head (Fig. 1). The full scan of the space within the field of view occurs during each missile rotation period. No complex, expensive and deceptive mechano-optical systems are required [6].

A disadvantage of this solution is the different rate of data acquisition depending on the distance a detector element to the optical axis increasing proportionally with the distance. In consequence the signal/noise ratio is worsening for more distant elements. This necessitates the use of better detectors with what results in increased costs of production.

A possible solution is the use of a dedicated simple two-dimensional detector array with a such distribution of elements that the worsening of signal/noise ratio for parts of image plane which are distant from optical axis can be compensated by Time Delay Integration (TDI) technique.

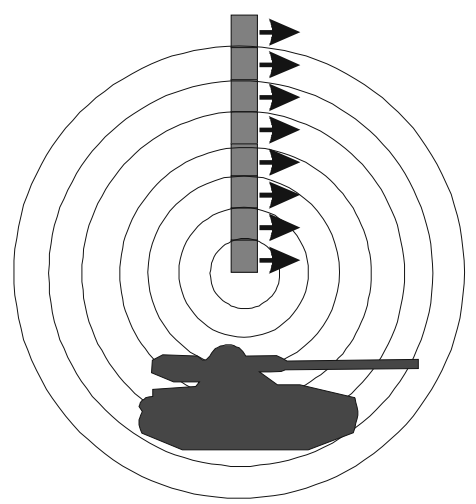

Fig. 1 The space scanning by missile rotation with a linear detector array

\section{The detection system}

\subsection{The idea of detector}

One possible implementation of the detector optimized for missile rotation based scan systems is a sector-shaped 2D array shown in Fig. 2.

The use of the two-dimensional array of detectors with such distribution of elements makes possible compensation of the signal/noise ratio worsening for the array elements which are distant to the optical axis. The proposed idea exploits an appropriate increase of detector elements number and application of TDI technique which consists in scanning of image plane by many elements of detector simultaneously and integration of measured signals from different detection elements along the scan direction with the appropriate delays in time [7]. 


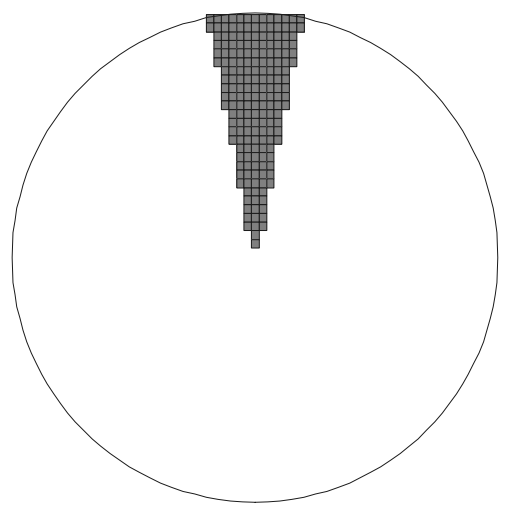

Fig. 2 The schematic drawing of the sector-shaped 2D detector array

\subsection{The probability of target detection}

The present standard for probability of target detection is based on the Johnson method [8] which distinguishes three classes: detection, recognition and identification. The detection takes place when the object will appear in the observed image. The recognition allows for a more exact qualification of an object - tank, car and man. The identification permits to fix exactly all basic characteristics and external features of an object and to qualify faultlessly its type, affiliation and moving speed. The Johnson method was originally worked out for testing the visual estimation of bar pattern discrimination. Its relies on replacement of an observed target by a four-bar tester and then on increasing of the distance between observer and tester as far as the discrimination of streaks is still possible. In case of optical-electronic systems the Johnson criterion is used for discrimination of two-dimensional targets (Table 1).

Table 1. The two-dimensional discrimination of target

\begin{tabular}{|c|c|}
\hline Class of discrimination & Cycles number of test \\
\hline Detection & 0.75 \\
\hline Recognition & 3.0 \\
\hline Identification & 6.0 \\
\hline
\end{tabular}

The required size of a detector is

where:

$$
l=\frac{\sqrt{h w}}{2 \frac{n}{L}} \cdot f
$$

$h$ - height, $w$ - width of target, $n$ - cycles number of test on critical size, $L$ - distance to target, $f$ - focal length.

According to equation (1), the required size of a detector for a specific target discrimination class and probabilities depends on the distance to the target, its critical size and testing cycles number. The coefficient of probability depending on a cycle's number is given by [8]: 


$$
P(N)=\frac{\left(\frac{N}{N_{50}}\right)^{E}}{1+\left(\frac{N}{N}\right)^{E}} \quad E=2.7 \cdot 0.7\left(\frac{N}{N_{50}}\right)
$$

where:

$N$ - is the cycles number, and $N_{50}$ - is the cycles number for $50 \%$ probability.

Table 2 show the results of target discrimination probability calculations, a temporary field of view and the detector size depending on the cycles number for the critical size of the target.

Table 2. The instantaneous field of view DAS, detector length and probability of detection, recognition and identification as a function of cycles number for the critical size of an object

\begin{tabular}{|c|c|c|c|c|c|}
\hline Cycles & DAS & \multirow{2}{*}{ L $[\mathrm{mm}]$} & \multicolumn{3}{|c|}{ Probability } \\
\cline { 4 - 6 } & & detection & recognition & identification \\
\hline 1 & 2.61 & 0.261 & $74 \%$ & $3.8 \%$ & $0.06 \%$ \\
\hline 2 & 1.31 & 0.131 & $97 \%$ & $23 \%$ & $4.3 \%$ \\
\hline 3 & 0.87 & 0.087 & $99 \%$ & $50 \%$ & $12 \%$ \\
\hline 4 & 0.65 & 0.065 & $99 \%$ & $70 \%$ & $24 \%$ \\
\hline 5 & 0.52 & 0.052 & $99 \%$ & $82 \%$ & $37 \%$ \\
\hline 6 & 0.44 & 0.043 & $99 \%$ & $88 \%$ & $50 \%$ \\
\hline
\end{tabular}

\subsection{The thermal and spatial resolution of the missile rotation based imaging system}

Sensitivity is one of the most important characteristics of IR sensor. For less distant targets which can be treated as extensive objects, the basic characterisation of sensitivity is the thermal resolution usually defined as the Noise Equivalent Difference (NETD) [9]:

$$
N E T D=\frac{4 F^{2} \Delta f^{1 / 2}}{A_{d}^{1 / 2} M^{*}}
$$

where:

$F$ - is the focal ratio, $\Delta f$ - electric bandwidth, $A_{d}$ - area of detector and $M$ - thermal fugure of merit of a detector.

$$
M^{*}=\int_{\lambda} D *(\lambda) \frac{\partial M(\lambda, T)}{\partial T} d \lambda
$$

where:

$D^{*}(\lambda)$ is the spectral detectivity, $M(\lambda, T)$ - spectral exitance determined by the Planck law.

Thus temperature resolution depends on optics, detector size and detectivity and matching of its spectral response to the temperature derivative of spectral exitance of the object. The analysis of expression (3) shows that increase of thermal 
resolution for the same field of view and scanning time leads to a proportional deterioration of spatial resolution.

The noise bandwidth for every active element of detector array can be specified by the formula:

$$
\Delta f=\frac{N \omega}{2}
$$

where:

$\omega$ - angular velocity of rotation, $N$ - number of an orbit.

This dependence shows that the noise bandwidth increasing proportionally to number of an orbit. A comparison is made between for the simple 32 - elements linear array (Fig. 1) and for arrays where the number of elements on every orbit is equal to the number of an orbit (Fig. 2). The assumed period of missile rotation is $0.1 \mathrm{~s}$, detector area $200 \times 200 \mu \mathrm{m}$, focal length $\mathrm{f}=100 \mathrm{~mm}$ and $\mathrm{f}$-number $F=1.5$. Then the instantaneous field of view will be $2 \times 2$ mrad. The equivalent noise bandwidth is then $1 \mathrm{kHz}$ for the detectors groups on each of the 32 orbits.

We have calculated detectivities of detector elements operating in the 7.8-10 $\mu \mathrm{m}$ spectral range that is required for $0.1 \mathrm{~K}$ thermal resolution in imaging of $\approx 300 \mathrm{~K}$ objects. As the calculation show, detectivities of $1.3 \times 10^{9}$ and $2.3 \times 10^{8}$ are necessary for the simple linear array the sector array, respectively.

Recent studies show, that the required performance can be obtained with an array uncooled $\mathrm{Hg}_{1-\mathrm{x}} \mathrm{Cd}_{\mathrm{x}} \mathrm{Te}$ photodetectors [9-15].

\section{Detector}

As calculations show, the use of missile rotation for space scanning requires detectors with bandwidth of at least $1 \mathrm{kHz}$. This means that uncooled thermal detectors (microbolometers or pyroelectric) are too slow for such applications. In contrast, any photodetectors are fast enough, but achievement of the required performance without cryogenic cooling present a substantional challenge.

Recently, a Warsaw based Polish company, VIGO SYSTEM S.A. has developed MWIR (3-5.5 $\mu \mathrm{m})$ and LWIR $(7.8-10 \mu \mathrm{m}) \mathrm{Hg}_{1-\mathrm{x}} \mathrm{Cd}_{\mathrm{x}}$ Te photodetector arrays dedicated to the missile rotation based ATR imaging systems [9-15]. The device operate at ambient temperature or at temperatures achievable with simple Peltier or evaporative coolers. Photoconductive devices have been initially used. Suffering from a high low frequency noise they were not particularly useful for the missile rotation based imaging with relatively low operating frequencies and they were eventually replaced by unbiased photovoltaic devices that are essentially free from the flicker noise.

The practical devices are based on heterostructures grown by combination of the ISOVPE and MOCVD epitaxial techniques. The performance of the device have been optimized by the use of band gap engineered $\mathrm{Hg}_{1-\mathrm{x}} \mathrm{Cd}_{\mathrm{x}} \mathrm{Te}$ multilayer heterostructures with complex 3D band gap and doping level profiles in which the electrical that integrate both the optical and photoelectrical functions leading to superior detection characteristics without need for cryogenic cooling [14-15].

\section{Conclusions}

Homing heads of rotating missiles can be based on simple electrooptical systems that employ missile rotation for space scanning. Special formats of detector arrays 
are proposed that compensate for increasing bandwidth of detector elements at larger distances from the axis of the optical system.

Results of theoretical analyses and experimental research show that homing heads of rotating missiles with uncooled IR photodetectors can achieve useful temperature and spatial resolutions.

The practical $\mathrm{Hg}_{1-\mathrm{x}} \mathrm{Cd}_{\mathrm{x}} \mathrm{Te}$ photovoltaci detectors that can be used for the missile rotation based imaging system have been developed and characterized.

\section{Acknowledgements}

The work was performed under the Polish Committee for Scientific Investigation Grant No. KBN 0T00B00626.

\section{REFERENCES}

[1] J.W. Sherman „Automatic target recognition systems”, The Infrared \& Electrooptical Systems Handbook, SPIE, Bellingham (1993)

[2] T.L. Williams, J.H. Ludlow "Introduction to military thermal imaging" SIRA Technology Center, London (1994)

[3] J. Piotrowski, M. Grudzień, K. Pawelek, S. Stepniak and W. Swiderski „IR detectors for smart ammunition" (in Polish) PTUIR, 69, pp. 73-84 (1999)

[4] R. Hudson and J. Hudson "Infrared Detectors" Hutchinson and Ross, Wiley (1975)

[5] The Infrared \& Electrooptical Systems Handbook, SPIE, Bellingham (1993)

[6] J. Piotrowski, D. Szabra „IR detectors of homing head for rotating missiles of smart munition" (in Polish) (in print)

[7] J. Piotrowski, D. Szabra "Conception of advanced IR detector for homing head of rotating missiles" (in Polish) PTU, 84, pp. 31-36 (2002)

[8] G. C. Holst „Electro-Optical Imaging System Performance”

[9] G. Gaussorges "La thermographic Infrarouge" Lavoiser, Paris (1984)

[10] J. Piotrowski and M. Razeghi, „Improved performance of IR photodetectors with 3D gap engineering", Proc. SPIE, 2397, pp. 180-192, 1995

[11] J. Piotrowski, „Suppression of Auger generation as the way to perfect detection of infrared radiation", PTU, 82, No 2, pp. 57-68, 2002

[12] P. Brzozowski, "Analysis of special applications of uncooled MWIRA and LWIR photodetectors" Thesis (in Polish). Warsaw Technical University. 2003

[13] J. Piotrowski and P. Brzozowski, „Uncooled IR photodetectors for smart munitions" PTUiR, 72, pp. 29-50, 2000

[14] Final report of Grant No. KBN 0T00B00626

[15] J. Piotrowski, "Uncooled operation of IR photodetectors", Opto-Electronics Rev. 12, 111-122 (2004). 